\title{
N2b Stage Finding
}

National Cancer Institute

\section{Source}

National Cancer Institute. N2b Stage Finding. NCI Thesaurus. Code C48712.

A general term that refers to a TNM finding of cancer metastases in several regional lymph nodes. The definition of N2b TNM finding depends on the specific type of cancer that it refers to; for example, for breast cancer it refers to metastases only in clinically detected ipsilateral internal mammary nodes and in the absence of clinically evident level I, II axillary lymph node metastases; for cutaneous melanoma it refers to macrometastases in 2-3 regional lymph nodes; for colorectal cancer it refers to metastases in seven or more regional lymph nodes. 